\title{
Preditores da Síndrome de Burnout em professores
}

\author{
Síndrome de Burnout em professores
}

\author{
Mary Sandra Carlotto \\ Sheila Gonçalves Câmara
}

\section{Resumo}

O objetivo deste estudo foi identificar os preditores da Síndrome de Burnout em 563 professores de instituições educacionais particulares da região metropolitana de Porto Alegre - RS. Foram utilizados um questionário elaborado para levantamento de variáveis demográficas e profissionais, o Maslach Burnout Inventory, o Job Diagnostic Survey e o Questionário de Satisfação no Trabalho S20/23. Os resultados evidenciam que variáveis relacionadas ao contexto laboral predominam no modelo explicativo de Burnout em professores em ambos os grupos.

Palavras-Chave: Professores; Síndrome de Burnout; Preditores.

\section{Predictors of Burnout Syndrome in teachers}

\begin{abstract}
The aim of this study was to identify the predictors for the Burnout Syndrome in 563 teachers from private educational institutions of the Porto Alegre's metropolitan area. A specific questionnaire was developed in order to evaluate demographic and professional variables. The Maslach Burnout Inventory, the Job Diagnostic Survey and the Questionnaire of Satisfaction were also used. Results showed a predominance of variables associated to the work enviroment in the explicative model of burnout for both groups, although with a different configuration in each group.

Keywords: Teachers; Burnout Syndrome; Predictors.
\end{abstract}

\section{Predictores del Síndrome de Burnout en profesores}

\section{Resumen}

El objetivo de este estudio fue identificar los predictores del Síndrome de Burnout en 563 profesores de instituciones educacionales privadas de la región metropolitana de Porto Alegre - RS. Fueron utilizados un cuestionario elaborado para verificar las informaciones relativas a variables demográficas y profesionales, el Maslach Burnout Inventory, el Job Diagnostic Survey y el Questionário de Satisfação no Trabalho S20/23. Los resultados mostraron que variables relacionadas al contexto laboral predominan en el modelo explicativo de Burnout en profesores de los dos grupos.

Palabras clave: profesores, síndrome de Burnout, predictores. 


\section{Introdução}

Novos cenários têm sido definidos, em um mundo globalizado com o capitalismo mundial integrado, produção de novas tecnologias, aceleração de produção, trabalho e trabalhadores flexíveis e polivalentes, acompanhados de trabalhadores precarizados e excluídos das inovações. Estas mudanças se refletem na reestruturação e reforma dos sistemas educativos, afetando o quadro docente, o trabalho que desempenham e as relações profissionais e sociais (Gomes \& Brito, 2006) com visíveis repercussões na qualidade de vida no trabalho e saúde do professor. As principais mudanças ocorridas relacionam-se à redução da amplitude de sua atuação, isto é, tarefas de alto nível transformadas em rotinas, existindo mais subserviência a um conjunto de aspectos burocráticos. O professor possui atualmente menos tempo para executar o trabalho, menos tempo para atualização profissional, lazer, convívio social e reduzidas oportunidades de trabalho criativo. Há uma maior diversificação de responsabilidade com distanciamento entre a execução, realizada pelos professores, e o planejamento das políticas que norteiam seu trabaIho, geralmente elaboradas por outras pessoas (Kelchtermans, 1999).

Professores, no entanto, não pertencem a um grupo homogêneo, pois dentro desta categorização há diferenças nas funções e atribuições. Sendo assim, o trabalho desenvolvido também apresenta diferentes desafios, demandas e recompensas dependendo de variáveis como nível de ensino, tipo de instituição, pública ou privada, urbana ou rural, faixa etária dos alunos, contexto social onde está inserida, entre outras (Guglielmi \& Tatrow, 1998).

Nas escolas, o professor, além de atender a classes, deve fazer trabalhos administrativos, planejar, reciclar-se, investigar e orientar individualmente os alunos. Ele deve, também, organizar atividades extraescolares, participar de reuniões pedagógicas e de coordenação, seminários, conselhos de classe, efetuar processos de recuperação, preenchimento de relatórios bimestrais e individuais relativos às dificuldades de aprendizagem de alunos e, muitas vezes, cuidar do patrimônio, material, recreios e locais de re- feições (Esteve, 1999; Nacarato, Varani \& Carvalho, 2000; Schnetzler, 2000; Woods, 1999). Nas instituições de ensino superior, esse profissional deve conciliar atividades de ensino, pesquisa e extensão, atendendo questões relacionadas à produção científica, além de ter, também, que executar as administrativas. Segundo Franco (200I), o professor do ensino superior está permanentemente sob um crivo crítico, desde o ingresso na carreira, através de avaliações sistemáticas para a ascensão profissional, da submissão de trabalhos em eventos, da apresentação de projetos e de relatórios de atividades e de pesquisa.

Assim, acredita-se que a organização do trabalho do professor, atualmente, possui características que o expõem a fatores estressantes que, se persistentes, podem levar à Síndrome de Burnout (Guglielmi \& Tatrow, 1998). De acordo com Maslach, Schaufeli, e Leiter (200I), Burnout é um fenômeno psicossocial que surge como uma resposta crônica aos estressores interpessoais ocorridos na situação de trabalho, sendo constituído de três dimensões relacionadas, mas independentes: Exaustão Emocional, Despersonalização e sentimento de Baixa Realização Profissional (Maslach \& Jackson, 198I; Maslach, 1993; Maslach, Schaufeli \& Leiter, 200I; Benevides-Pereira, 2002). A Exaustão Emocional é caracterizada pela falta ou carência de energia, entusiasmo e por sentimento de esgotamento de recursos. A Despersonalização faz com que o profissional passe a tratar os clientes, colegas e a organização como objetos. Já a Baixa Realização Profissional caracteriza-se por uma tendência do trabalhador em se auto-avaliar de forma negativa, sentindo-se infeliz e insatisfeito com seu desenvolvimento profissional.

Burnout tem atingido várias profissões, mas tem seu foco de estudo especialmente vinculado à área de ensino e serviços de saúde por serem atividades que envolvem intenso contato com pessoas (Maslach \& Jackson, 1985; Maslach \& Leiter, 1997). No caso de sua ocorrência em professores, esse fenômeno afeta - ambiente educacional e interfere na obtenção dos objetivos pedagógicos, levando os professores a um processo de alienação, cinismo, apatia, problemas de saúde e intenção de abandonar a profissão (Guglielmi \& Tatrow, 1998). 
Estudos têm evidenciado que as variáveis que afetam Burnout em professores primários e secundários são diferentes das que afetam os professores universitários. A exaustão emocional é a variável central encontrada em professores de ensino fundamental e médio, não ocorrendo o mesmo resultado com professores universitários (Byrne, 1991; Dillon \& Tanner, 1995).

Frente ao exposto, esta investigação buscou identificar se variáveis demográficas, laborais e dimensões de Características de Cargo, Estados Psicológicos Críticos, Resultados do Trabalho e Satisfação no Trabalho predizem (reduzem ou incrementam) a Síndrome de Burnout em professores universitários e não universitários, verificando se os preditores se apresentam de forma diferenciada. Para atender a este objetivo, optou-se por um estudo de caráter observacional analítico de corte transversal (Grimes \& Schulz, 2002).

\section{Método}

\section{Participantes}

A amostra constituiu - se de 563 professores, 280 de ensino universitário e 283 de ensino não universitário que exercem atividade docente em instituições particulares localizadas na região metropolitana de Porto Alegre - RS. O estudo foi realizado em oito escolas e uma universidade.

Os professores que constituem o grupo estudado são, em sua maioria, mulheres $(69,4 \%)$, casados (62\%), com filhos $(58,1 \%)$ e situam-se na faixa etária de 30 a 50 anos (68,9\%). Ambos os grupos, universitários e não-universitários, mantém as características acima descritas; no entanto, apresentam-se de forma diferenciada na sua distribuição por sexo e faixa etária.

No grupo de professores universitários, percebese uma tendência ao equilíbrio entre homens e mulheres, uma vez que $55,4 \%$ pertencem ao sexo feminino e 43,2\% ao masculino. Já em professores não-universitários há uma predominância de profissionais do sexo feminino $(83,4 \%)$. A média de idade desse grupo é de 36 anos $(D P=9,36)$. Verifica-se que o número de pro- fessores não-universitários, com menos de 30 anos, representa um percentual maior que o dobro dos não universitários $(25,8 \%)$. E na faixa etária de mais de 50 anos, os professores universitários apresentam percentuais próximos ao dobro dos não universitários (13,6\%), com uma média de idade de 40 anos e 3 meses $(D P=8,68)$. Com relação às características laborais, pouco mais da metade dos professores desenvolve suas atividades com uma carga horária que varia de vinte a mais de quarenta horas semanais $(52,6 \%)$ e, geralmente, em dois turnos de trabalho (47,9\%).A maior parte do grupo $(59,6 \%)$ concilia seu trabalho com atividades extra-institucionais de forma autônoma ou vinculada à outra organização de trabaIho. Os participantes possuem um tempo médio de atuação docente no ensino geral de 12 anos e 2 meses, de ensino em nível universitário de 8 anos e 2 meses e de ensino na universidade onde a pesquisa foi realizada de 5 anos el 0 meses.

No grupo de professores de ensino não universitário, o nível de maior concentração é o Ensino Fundamental $(44,2 \%)$, seguido pela combinação de exercício deste nível com o Ensino Médio (30\%). O turno da manhã concentra a maioria dos professores $(77,7 \%)$. O trabalho em um $(44,2 \%)$ ou dois turnos $(44,4 \%)$ é a predominância nesta categoria de ensino. Mais da metade do grupo desenvolve suas atividades numa carga horária acima de 20 horas $(55,8 \%)$. Deste grupo, $42 \%$ atuam somente em uma escola, $38,1 \%$ trabalham em outra instituição de ensino e $16,2 \%$ desenvolvem outro tipo de trabalho profissional. Possuem 12 anos e 10 meses de experiência no ensino e 6 anos e 10 meses na escola atual.

\section{Instrumentos}

Para operacionalizar os objetivos desta investigação utilizou-se quatro instrumentos auto-aplicáveis:

Questionário para levantamento de dados

Os dados demográficos coletados foram (sexo, idade, estado civil, filhos) e os laborais referiram-se (tempo de experiência docente, tempo de trabalho na universidade ou na escola, carga-horária semanal, número de alunos/dia, número de horas de ensino, pesquisa e extensão) por meio do questionamento elaborado especificamente para responder aos 
objetivos do estudo, tendo como base principal o referencial teórico sobre a Síndrome de Burnout em professores. Para variáveis qualitativas foram utilizadas questões fechadas e para as quantitativas, questões abertas.

Maslach Burnout Inventory (Tradução para a língua portuguesa validada por Lautert, 1995).

O inventário avalia como o trabalhador vivencia seu trabalho, de acordo com três dimensões: Exaustão Emocional (9 itens), Desenvolvimento Pessoal (8 itens) e Despersonalização (5 itens). O questionário totaliza 22 itens que indicam a freqüência das respostas com uma escala de pontuação que varia de I a 7 (Maslach \& Jackson, 198I). Para este estudo foi utilizada a pontuação de I a 5, empregada e validada no Brasil por Tamayo (1997), onde a opção nunca pontua I e todos os dias pontua 5. Segundo Maslach e Jackson (198I, 1986), a consistência interna das três dimensões do inventário é satisfatória, pois apresenta um alfa de Cronbach que vai desde 0,7l até 0,90 e os coeficientes de teste e re-teste vão de 0,60 a 0,80 em períodos de até um mês. Um estudo realizado por Carlotto e Câmara (2004) indica que a versão brasileira do $\mathrm{MBI}$ apresenta os requisitos necessários em termos de consistência interna e validade fatorial para ser utilizada na avaliação da Síndrome de Burnout em professores em nossa realidade

Job Diagnostic Survey (Hackman \& Oldhan, 1980)

Avalia as Características de Cargo com foco na motivação para o trabalho a partir de variáveis de conteúdo - Significado percebido do trabalho, Responsabilidade percebida, Conhecimento dos resultados do trabalho, Variedade de habilidades utilizadas, Identidade da tarefa, Significado da tarefa, Autonomia, Feedback intrínseco, Feedback extrínseco e Inter-relacionamento - e variáveis de contexto - Possibilidade de crescimento, Segurança no trabalho, Compensação, Ambiente social e Supervisão. As dimensões avaliadas são Características básicas do cargo, Estados psicológicos, Reações afetivas e Satisfação frente a determinados fatores de contexto do cargo e a lntensidade da necessidade de auto-realização. Para o presente estudo foi utilizada a adaptação espanhola do questionário de Fuertes, Monduate e Fortea (1996), Forma A. O questionário é constituído de sete seções, distribuído em 82 itens: seção I (7 itens), seção 2 (14 itens), seção 3 (I5 itens), seção 4 (14 itens), seção 5 (I0 itens), seção 6 (II itens) e seção 7 ( 12 itens). A consistência interna do instrumento é de 0,78 (Fuertes, Monduate \& Fortea, 1996).

Questionário de Satisfação no Trabalho (Meliá e colaboradores, 1984).

Esta versão possui 23 itens distribuídos em cinco fatores: I. Satisfação com a Supervisão (6 itens), II. Satisfação com o Ambiente Físico de Trabalho (5 itens), III. Satisfação com Benefícios e Políticas da Organização (5 itens), IV. Satisfação Intrínseca do Trabalho (4 itens) e V. Satisfação com a Participação (3 itens). A consistência interna do questionário é de 0,92 . Os cinco fatores apresentaram índices de 0,89 , 0,8I, 0,76, 0,80, 0,78. (Meliá \& Peiró, 1989). O Questionário foi traduzido para o português por dois psicólogos e a tradução obtida foi novamente traduzida (back-translation) por outros dois psicólogos com domínio da língua espanhola. Segundo Carlotto (2005), a versão brasileira do S20/23 apresentou os requisitos necessários em termos de consistência interna e validade fatorial para ser utilizada em professores com nossa realidade. Os cinco fatores apresentaram alfas de $0,9 \mathrm{I}, 0,86,0,82,0,78,0,83$.

\section{Procedimento}

Para a coleta de dados, um contato com a direção das instituições de ensino foi primeiramente realizado e, em seguida, apresentado o objetivo do estudo a fim de obter a autorização e o apoio para a aplicação dos instrumentos. Estes foram entregues aos professores em seu Diário de Classe, sendo a coleta realizada por intermédio de urnas colocadas no Apoio Docente ou na Sala dos Professores. Os instrumentos foram recolhidos uma semana após a retirada dos diários. A aplicação foi realizada no mês de agosto na universidade e outubro de 2002 nas escolas, meses que não ocorriam provas ou outros eventos importantes nas instituições relacionadas ao corpo docente e que poderiam dificultar o preenchimento do instrumento. Os procedimentos éticos foram realizados, conforme a legislação do Ministério da Saúde (1997), resolução 196 do Conselho Nacional de Saúde (CNS). Aos professores e diretores das instituições de ensi- 
no foi esclarecido tratar-se de uma pesquisa sem quaisquer efeitos avaliativos individuais e/ou institucionais e que as respostas seriam anônimas e confidenciais. $O$ Banco de Dados foi digitado e posteriormente analisado em pacote estatístico. As médias e o desvio-padrão das dimensões de Burnout foram calculadas e também realizada a análise de regressão linear múltipla a fim de identificar os preditores de cada dimensão, em cada grupo.

\section{Resultados}

Os resultados indicam, nos dois grupos, maior índice em Exaustão Emocional, seguido pela dimensão de Baixa Realização Profissional e menor índice em Despersonalização (Tabela I). apontam para um modelo preditor composto pelas variáveis: Satisfação com o Pagamento (SAT-P) e a Idade. Estas, conjuntamente, explicam $46,4 \%$ da variabilidade da Exaustão Emocional. A variável com maior poder explicativo refere-se à Satisfação com o Pagamento com $35 \%$ de variância.

Com relação à Despersonalização, o modelo final explicou $57,8 \%$ da variabilidade, sendo detectadas como preditoras as variáveis Horas de Pesquisa (HP) e Conhecimento dos Resultados do Trabalho (CR), ainda que se verifique que a variável com maior poder explicativo é a carga horária de pesquisa com $49 \%$.

Por fim, a análise de regressão múltipla para a VD Baixa Realização Profissional, revelou como preditoras as variáveis: Satisfação com o Crescimento (SAT-A), Necessidade de Auto-realização (NE), Satisfação Geral (SAT-G), Horas de Pesquisa (HP), Significado da

Tabela I. Estatísticas descritivas das dimensões de Burnout

\begin{tabular}{|c|c|c|c|c|}
\hline \multirow{3}{*}{ Dimensões } & \multicolumn{4}{|c|}{ Professores } \\
\hline & \multicolumn{2}{|c|}{ Universitários } & \multicolumn{2}{|c|}{ Não universitários } \\
\hline & M & $\mathrm{DP}$ & $\mathrm{M}$ & DP \\
\hline Exaustão Emocional & 2,21 & 0,67 & 2,34 & 0,65 \\
\hline Despersonalização & 1,48 & 0,51 & 1,49 & 0,46 \\
\hline Baixa Realização Profissional & 1,53 & 0,46 & 1,67 & 0,55 \\
\hline
\end{tabular}

Três análises de regressão múltipla por grupo de professores (universitários e não- universitários) foram realizadas pelo método stepwise, tendo cada uma como variável dependente a dimensão do $\mathrm{MBI}$ correspondente, ou seja: Exaustão Emocional, Despersonalização e Baixa Realização Profissional, e como variáveis independentes as referentes a sexo, idade, número de filhos, tempo de experiência docente, tempo de trabalho na universidade ou na escola, carga-horária semanal, número de alunos/dia, número de horas de ensino, pesquisa e extensão; dimensões de Características de Cargo, Estados Psicológicos Críticos, Resultados do Trabalho e Satisfação no Trabalho.

Os resultados obtidos no grupo de professores universitários na dimensão de Exaustão Emocional
Tarefa (ST) e Satisfação Intrínseca com a Tarefa (SIT). A com maior poder explicativo foi a Satisfação com Crescimento com $47 \%$, tendo o modelo final explicado $84,2 \%$ da variabilidade total desta dimensão.

Os resultados evidenciam relação inversa entre as variáveis, Satisfação com o Pagamento e Idade com Exaustão Emocional. Os profissionais com menos idade e com menor satisfação com sua remuneração apresentam maior exaustão emocional. A variável Horas de Pesquisa apresenta relação positiva, indicando que quanto mais horas dedicadas a esta atividade, maior o sentimento de distanciamento pessoal. O Conhecimento dos Resultados obtidos com o trabalho se relaciona negativamente, revelando sua potencialidade para diminuir a Despersonalização. Com relação à Baixa Realização Profissional, a Satisfação com o Crescimen- 
to, a Satisfação Geral, o Significado da Tarefa e a Satisfação Intrínseca com o trabalho apresentaram relação inversa, isto é, influenciam para a diminuição do sentimento de baixa realização profissional. Já a Necessidade de Auto-realização e as horas de pesquisa evidenciaram relação positiva sinalizando que quanto maior a necessidade de auto-realização e as horas dedicadas à pesquisa, maior é o sentimento de baixa realização profissional (Tabela 2). novamente a Satisfação com o Crescimento com $17,9 \%$.

Por fim, a análise de regressão múltipla para a VD Baixa Realização Profissional, revelou como preditoras as variáveis, Satisfação com o Crescimento e Contato Social, tendo a primeira maior poder explicativo, com $16 \%$. O modelo final é explicado com $21,1 \%$ da variabilidade total desta dimensão. Os resultados indicam existir relação negativa entre a Satisfação com

Tabela 2. Regressão múltipla no grupo de professores universitários

\begin{tabular}{lccccc}
\hline Variáveis & $\mathbf{R}$ & $\mathbf{R}^{2}$ & Beta & $t$ & $p$ \\
\hline Exaustão Emocional & & & & & \\
1.SAT-P (Satisfação com o Pagamento) & 0,592 & 0,350 & $-0,502$ & $-3,311$ & 0,003 \\
2.IDADE & 0,681 & 0,464 & $-0,348$ & $-2,297$ & 0,030 \\
\hline Despersonalização & & & & & \\
1.HP (Horas de Pesquisa) & 0,700 & 0,490 & 0,670 & 5,123 & 0,000 \\
2.CR (Conhecimento dos Resultados) & 0,760 & 0,578 & $-0,297$ & $-2,272$ & 0,032 \\
\hline Baixa Realização Profissional & & & & & \\
1.SAT-A (Satisfação com o Crescimento) & 0,690 & 0,476 & $-0,443$ & $-3,761$ & 0,001 \\
2.NE (Necessidade de Auto-realização) & 0,770 & 0,592 & 0,385 & 3,475 & 0,002 \\
3.SAT-G (Satisfação Geral) & 0,820 & 0,673 & $-0,308$ & $-2,711$ & 0,013 \\
4.HP (Horas de Pesquisa) & 0,861 & 0,742 & 0,339 & 3,640 & 0,002 \\
5.ST (Significado da Tarefa) & 0,896 & 0,803 & $-0,278$ & $-2,897$ & 0,009 \\
6.SIT (Satisfação com o Trabalho) & 0,917 & 0,842 & $-0,228$ & $-2,274$ & 0,034 \\
\hline
\end{tabular}

Em professores não-universitários, no modelo final para Exaustão Emocional, duas variáveis apresentaram poder explicativo: Satisfação com o Crescimento Profissional (SAT-A) e Potencial Motivacional do Cargo (PM). Estas, conjuntamente, explicam 25,5\% da variabilidade da exaustão, sendo que a de maior poder explicativo foi a Satisfação com o Crescimento com $20,8 \%$.

Com relação à Despersonalização, o modelo final explicou $32,8 \%$ sendo elencadas como preditoras as variáveis: Satisfação com o Crescimento Profissional (SAT-A), a Variedade de Habilidades (VH) e Satisfação Intrínseca com a Tarefa (SIT), ainda que se constate que a variável com maior poder explicativo é
- Crescimento e as dimensões de Burnout, indicando que esta variável possui capacidade de reduzir suas dimensões. Já o Potencial Motivacional do Cargo apresenta relação positiva revelando poder para aumentar a Exaustão Emocional. A Variedade de Habilidades indica direcionalidade positiva, revelando que quanto mais diversificada a tarefa desenvolvida, maior a Despersonalização. Já a Satisfação Intrínseca revela direcionalidade inversa com a Despersonalização, quanto maior a satisfação com o trabalho, menor o sentimento de despersonalização. $O$ mesmo ocorre com relação ao Contato Social, evidenciando que quanto maior o contato social, menor o sentimento de despersonalização (Tabela 3). 
Tabela 3. Regressão múltipla no grupo de professores não-universitários

\begin{tabular}{|c|c|c|c|c|c|}
\hline Variáveis & $\mathbf{R}$ & $\mathbf{R}^{2}$ & Beta & $t$ & $p$ \\
\hline \multicolumn{6}{|l|}{ Exaustão Emocional } \\
\hline 1. SAT-A (Satisfação com o Crescimento) & 0,456 & 0,208 & $-0,472$ & $-4,690$ & 0,000 \\
\hline 2. PM (Potencial Motivacional do Cargo) & 0,505 & 0,255 & 0,217 & 2,153 & 0,035 \\
\hline \multicolumn{6}{|l|}{ Despersonalização } \\
\hline 1.SAT-A(Satisfação com o Crescimento) & 0,423 & 0,179 & $-0,354$ & $-3,457$ & 0,001 \\
\hline 2.VH (Variedade de Habilidades) & 0,488 & 0,238 & 0,316 & 3,175 & 0,002 \\
\hline $\begin{array}{l}\text { 3.SIT (Satisfação Intrínseca com o } \\
\text { Trabalho) }\end{array}$ & 0,573 & 0,328 & $-0,327$ & $-3,117$ & 0,003 \\
\hline \multicolumn{6}{|l|}{ Baixa Realização Profissional } \\
\hline 1.SAT-A (Satisfação com o Crescimento) & 0,400 & 0,160 & $-0,414$ & $-4,004$ & 0,000 \\
\hline 2.CS (Contato Social) & 0,459 & 0,211 & $-0,226$ & $-2,188$ & 0,032 \\
\hline
\end{tabular}

\section{Discussão}

A análise de preditores indica predominância das variáveis relacionadas ao trabalho em ambos os grupos. Este resultado parece confirmar estudos que apontam serem as variáveis laborais as mais relacionadas ao Burnout (Boada, Vallejo \& Agullo-Thomás, 2004; González-Gutiérrez \& colaboradores, 2004; Leiter \& Maslach, 1988; Maslach \& Leiter, 1997; Maslach \& Godberg, 1998; Maslach, Schaufeli, \& Leiter, 200I; McCulloch \& O'Brien, 1986). Este aspecto pode ser visto no poder explicativo conjunto das variáveis que influenciam as dimensões que compõem as Características do Cargo (Dimensões Básicas do Cargo, Estados Psicológicos Críticos, Resultados do Trabalho) e Satisfação no Trabalho, embora ocorram diferenças entre os grupos.

Em professores universitários, a variável de maior poder explicativo para a dimensão de Exaustão Emocional foi a Satisfação com o Pagamento. Na medida em que aumenta sua satisfação, diminui seu sentimento de desgaste. O professor universitário, segundo Lampert (1999), comparado com os docentes de escolas, é um profissional que tem maiores vantagens como melhores salários e condições de trabalho. Pode-se pensar que, por este grupo ter uma formação a qual necessita maior investimento, em termos de experiência profissional e titulação, cria expectati- vas, que se não atendidas, elevariam seu grau de desgaste emocional. Destaca-se que esta situação está mais presente nos professores mais jovens, que provavelmente possuem maiores expectativas, uma vez que a idade surge como segunda variável explicativa deste modelo.

Com relação à Despersonalização, a Carga Horária de Pesquisa foi a variável com maior poder explicativo. Sua elevação aumenta o sentimento de distanciamento com relação aos alunos. É importante referir que na universidade investigada há um limite de 8 horas semanais destinadas pela instituição para a realização de tal atividade. Paralelo as suas pesquisas, o docente realiza orientação de monografias com alunos, geralmente, com pouca experiência e conhecimento e, não raras vezes, sem uma maior identificação com tal atividade. Como a segunda variável explicativa, com direcionamento negativo, é o conhecimento dos resultados atingidos pelo trabalho, podese hipotetizar que os professores ao realizar a atividade de pesquisa, necessitam obter retorno sobre a mesma, principalmente em uma perspectiva institucional. O professor universitário em início de carreira busca, dentre outros aspectos, o reconhecimento acadêmico. De acordo com Byrne (1991), o desenvolvimento de pesquisas e a publicação científica são fatores geradores de Burnout em professores universitários. 
A Satisfação com o Crescimento Profissional apresentou o maior percentual explicativo da dimensão de Baixa Realização Profissional, com direcionamento inverso, sugerindo que os professores universitários investem na carreira acadêmica e, quando obtém satisfação com este processo de desenvolvimento, apresentam maiores índices de realização no trabalho. Esta situação se confirma na medida em que a variável necessidade de auto-realização entra no modelo como segunda variável explicativa.

Em professores não-universitários, a Satisfação com o Crescimento foi a variável de maior poder explicativo para as três dimensões de Burnout. Estar satisfeito com os rumos da carreira profissional é uma variável que atenua o Burnout (Anderson \& Iwanicki, 1984; Maslach, 1976). Este resultado sugere que este grupo profissional, por possuir menor reconhecimento social (Farber, 199I), volta-se para aspectos motivacionais intrínsecos. Pode-se também pensar que por atuarem em instituições de menor porte, possuem maior proximidade com gestores escolares, e, conseqüentemente, recebem maior feedback institucional.

Segundo Moreno, Garrosa e Benevides-Pereira (2003, p. 32), "os aspectos sociais, econômicos e culturais não são secundários ao problema do Burnout, são intrínsecos ao mesmo". Níveis similares de Burnout entre duas populações não indicam uma estrutura interna de Burnout similar, uma vez que os fatores etiológicos e preditivos, assim como suas conseqüências, podem ter configurações desiguais.

É importante estudar a cultura subjetiva do Burnout em diferentes populações, inclusive a de profissionais. A investigação realizada neste grupo indica peculiaridades, pois mesmo sendo uma mesma categoria profissional, apresentam-se marcadamente diferentes. Observa-se, através da literatura, que aspectos históricos da construção da profissão docente são diferenciados no contexto brasileiro. Há uma dicotomia social, cultural e econômica com relação a esta profissão. Professores universitários possuem uma representação social de maior status que professores de escolas.
Arce, Nakamura e Jólluskin (2003) referem que ainda há muitos resultados contraditórios em relação aos preditores de Burnout tanto com relação às condições de trabalho como em relação às variáveis demográficas e pessoais vinculadas ao mesmo. Assim, recomenda-se a realização de novos estudos, incluindo novas variáveis que possam ampliar o poder explicativo deste fenômeno psicossocial, que tem evidenciado importante implicação para a saúde mental dos professores.

\section{Referências}

Anderson, M. B. G., \& Iwanicki, E. F. (1984). Teacher motivation and its relationship to Burnout. Educational Administration Quaterly, 20, 109-132.

Arce, R., Nakamura, A. P., \& Jólluskin, G. (2003). Burnout: estudio del campo y nível de desempenho. Encuentros en Psicologia Social, I, 35-39.

Benevides-Pereira, A. M. T. (2002). Burnout: O processo de adoecer pelo trabalho. Em A. M. T. Benevides-Pereira (Org.), Burnout: Quando o trabalho ameaça o bem estar do trabalhador (pp.21-92). São Paulo: Casa do Psicólogo.

Boada, J., Vallejo, R. D., \& Agulló-Tomás, E. (2004). El Burnout y las manifestaciones psicosomáticas como consecuentes del clima organizacional y de la motivación laboral. Psicothema, 16, |25-|3|.

Byrne, B. (199I). Burnout: Investigating the impact of background variables for elementary, intermediate, secondary, and university educators. Teaching \& Teacher Education, 7, 197-209.

Carlotto, M. S. (2005). Síndrome de Burnout em professores de instituições particulares de ensino. (Tese de doutorado, Universidade de Santiago de Compostela, 2005). D.L.C, 202.

Carlotto, M. S., \& Câmara, S. G. (2004). Análise fatorial do Malasch Burnout Inventory (MBI) em uma amostra de professores de instituições particulares. Psicologia em Estudo, 9, 499-505.

Dillon, J. F., \& Tanner, G. R. (1995). Dimensions of career burnout among educators. Jornalism \& Mass Communication Educator, $50,4-14$.

Esteve, J. M. (1999). O mal-estar docente: A sala de aula e $a$ saúde dos professores. São Paulo: EDUSC. 
Farber, B. A. (1991). Crisis in education. Stress and burnout in the american teacher. São Francisco: Jossey-Bass Inc.

Franco, M. E. D. P. (200I). Comunidade de conhecimento, pesquisa e formação do professor do ensino superior. Em M. C. Morosini. (Org.), Docência universitária e os desafios da realidade nacional. Brasília: Plano.

Fuertes, M. F., Munduate, J. L., \& Fortea, B. M. A. (1996). Análisis y rediseño de puestos (Adaptação española del cuestionario Job Diagnostic Survey-JDS). Universitat Jaune I.

Gomes, L., \& Brito, J. (2006). Desafios e possibilidades ao trabalho docente e à sua relação com a saúde. Estudos e Pesquisas em Psicologia, 6(I) [On line]. Disponível em: <http:/ /www.revispsi.uerj.br/v6nl/artigos/PDF/v6nla05.pdf >

González-Gutiérrez, J. L., Moreno-Rodríguez, R., PeñacobaPuente, C., Alcocer-Costa, N., Alonso-Recio, L., BarcoCerro, P., \& Ardoy-Cuadros, J. (2004). Burnout in occupational therapy: an analysis focused on the level of individual and organizational consequences. Psychology in Spain, 8, 98-105.

Grimes, D. A., \& Shulz, K. F. (2002). An overview of clinical research: the lay of the land. The Lancet, 359, 57-6I.

Guglielmi, R. S., \& Tatrow, K. (1998). Occupational stress, burnout, and health in teachers: A methodological and theoretical analysis. Review of Educational Research, 68, 61-69.

Hackman, J. R., \& Oldhan, G. R. (1980). Work redesign. Massachustts: Addison - Wesley Reading.

Kelchtermans, G. (1999). Teaching career: Between burnout and fading away? Reflections from a narrative and biographical perspective. Em R.Vanderbergue \& $M$.

A. Huberman (Orgs.), Understanding and preventing teacher burnout: A source book of international practice and research (pp.I76-191). Cambridge: Cambridge University Press.

Lampert, E. (1999). Universidade, docência e globalização. Porto Alegre: Sulina.

Lautert, L. (1995). O desgaste profissional do enfermeiro. Tese de doutorado, Facultad de Psicologia, Universidade Pontificia de Salamanca, Salamanca.

Leiter, M. P., \& Maslach, C. (1988). The impact of interpersonal environment on burnout and organizational commitment. Journal of Organizational Behavior, 9, 297-308.

Maslach, C. (1976). Burned-out. Human Behavior, 5(9), 26-22.
Maslach, C. (1993). Burnout: A multidimensional perspective. Em W. B. Schaufeli, C. Maslach \& T. Marek (Orgs.), Professional burnout: Recent developments in theory and research (pp. 1932). New York: Taylor \& Francis.

Maslach, C., \& Goldberg, J. (1998). Prevention of burnout: News perspectives. Applied \& Preventive Psychology, 7, 63-74.

Maslach, C., \& Jackson, S. E. (198I). The measurement of experienced burnout. Journal of Ocuppational Behavior, 2, $99-113$.

Maslach, C., \& Jackson, S. E. (1985). The role of sex and family variables in burnout. Sex Roles, 12(7/8), 837-85I.

Maslach, C., \& Jackson, S. E. (1986). Maslach Burnout Inventory. (2nd. ed.). Palo Alto: Consulting Psychologist Press.

Maslach, C., \& Leiter, M. P. (1997). The truth about burnout: How organization cause, personal stress and what to do about lt. San Francisco: Jossey-Bass.

Maslach, C., Schaufeli, W. B., \& Leiter, M. P. (200I). Job burnout. Annual Review Psychology, 52, 397-422.

McCulloch, A., \& O’Brien, L. (1986). The organizational determinants of worker burnout. Children \& Youth Services Review, 8(2), 175-190.

Ministério da Saúde (1997). Conselho Nacional de Saúde. Diretrizes e normas para pesquisa envolvendo seres humanos. Resolução CNS 196/196. Brasília: Ministério da Saúde.

Meliá, J. L., \& Peiró, J. M. (1989). La medida de la satisfacción laboral en contextos organizacionales: El cuestionário de satisfacción S20/23. Psicologemas, 3, 59-74.

Moreno, B. J., Garrosa, E. H., \& Benevides-Pereira, A. M. (2003). Estudios transculturales del burnout. Los estudios transculturales Brasil-España. Encuentros de Psicologia Social, I, 3I-34.

Nacarato, A. M., Varani, A., \& Carvalho, V. (2000). O cotidiano do trabalho docente: palco, bastidores e trabalho invisível abrindo as cortinas. Em C. M. G Geraldi, D. Fiorentina \& E. M. A. Pereira. (Orgs.), Cartografias do trabalho docente (pp.73-104). Campinas: Mercado de Letras.

Schnetzler, R. P. (2000). Prefácio. Em C. M. G Geraldi, D. Fiorentina \& E. M. de A. Pereira (Orgs.), Cartografias do trabalho docente (pp.7-9). Campinas: Mercado de Letras.

Tamayo, R. M. (1997). Relação entre a síndrome de burnout e os valores organizacionais no pessoal de enfermagem de dois hospitais públicos. Dissertação de Mestrado, Instituto de Psicologia, Universidade de Brasília, Brasília. 
Woods, P. (1999). Intensification and stress in teaching. Em R. Vanderbergue \& M. A. Huberman (Orgs.), Understanding and preventing teacher burnout: A source book of international practice and research (pp. I I5-138). Cambridge: Cambridge University Press.
Recebido em: 14/10/2005

Revisado em: 26/04/2006

Aprovado em: 14/02/2007

Sobre as autoras:

Mary Sandra Carlotto (mscarlotto@superig.com.br) é Psicóloga, Mestre em Saúde Coletiva (ULBRA-RS), Doutora em Psicologia Social(USC/ES), Professora e Pesquisadora do Laboratório de Ensino e Pesquisa em Psicologia do Curso de Psicologia - ULBRA/ Canoas.

Sheila Gonçalves Câmara (scamara@via-rs.net) é Psicóloga, Mestre em Psicologia Social e da Personalidade e Doutora em Psicologia (PUCRS), Professora e Pesquisadora do Laboratório de Ensino e Pesquisa em Psicologia do Curso de Psicologia ULBRA/Canoas.

Endereço para correspondência

Sheila Gonçalves Câmara

Rua Edmundo Gardolinski, 70 - Bairro Boa Vista

90480- 130 - Porto Alegre-RS 